Research Article

\title{
Stochastic Linear Quadratic Control Problem on Time Scales
}

\author{
Yingjun Zhu (D) and Guangyan Jia (D) \\ Zhongtai Securities Institute for Financial Studies, Shandong University, Jinan 250100, China
}

Correspondence should be addressed to Guangyan Jia; jiagy@sdu.edu.cn

Received 14 June 2020; Revised 7 March 2021; Accepted 12 March 2021; Published 30 March 2021

Academic Editor: A. E. Matouk

Copyright (c) 2021 Yingjun Zhu and Guangyan Jia. This is an open access article distributed under the Creative Commons Attribution License, which permits unrestricted use, distribution, and reproduction in any medium, provided the original work is properly cited.

\begin{abstract}
This paper addresses a version of the stochastic linear quadratic control problem on time scales (S $\Delta \mathrm{LQ}$ ), which includes the discrete time and continuous time as special cases. Riccati equations on time scales are given, and the optimal control can be expressed as a linear state feedback. Furthermore, we present the uniqueness and existence of the solution to the Riccati equation on time scales. Furthermore, we give an example to illustrate the theoretical results.
\end{abstract}

\section{Introduction}

The linear quadratic control problem is one of the most important issues for the optimal control problem. It has received much attention [1-3] and has a wide range of applications in engineering and finance. Until now, the linear quadratic control problem is well understood from both continuous and discrete points of view. In this paper, the stochastic linear quadratic control problem is studied in the version of time scales.

Time scales were first introduced by Hilger [4] in 1988 in order to unite and extend the continuous and discrete analysis into a general framework. Time scales theory has been extensively studied in many works [5-10]. It is well known that the optimal control problem on time scales is an important field for both theory and applications. Since the calculus of variations on time scales was studied by Bohner [11], results on related topics and their applications have become more and more $[12,13]$. The existence of optimal control for the dynamic systems on time scales was considered in $[14,15]$. Subsequently, the maximum principle and dynamic programming in the time scale setting were studied [16, 17] for a deterministic dynamic system. In $[18,19]$, some results were obtained for the deterministic linear quadratic control problems on time scales. Recently, in Poulsen's Ph.D. thesis $[20,21]$, the authors developed an interesting theory of dynamic equations on time scales, in which the time scale is a discrete and stochastic one depending on a sequence of i.i.d. positive random variables, but the associated dynamic equation does not include stochastic terms; they also studied the associated control and stability problems.

In this paper, we are interested in the stochastic linear quadratic control problem on time scales ( $\mathrm{S} \Delta \mathrm{LQ}$ for short). Very similar to continuous and discrete cases, we can also obtain the associated Riccati equations (see [22, 23] for continuous and discrete cases) on time scales. Meanwhile, we discuss the existence and uniqueness of the solution to Riccati equations on time scales ( $\mathrm{R} \Delta \mathrm{E}$ for short). The difference from [24] is that the control system does not contain the expectation term, but includes the inhomogeneous term.

The organization of this paper is as follows. In Section 2, we introduce some preliminaries about the time scale theory and $S \triangle L Q$ problem. We will study the stochastic linear quadratic control problem on time scales and the associated Riccati equations on time scales in Section 3. Finally, an example is given.

\section{Preliminaries}

A time scale $\mathbb{T}$ is a nonempty closed subset of the real numbers $\mathbb{R}$, and we denote $[0, T]_{\mathbb{T}}=[0, T] \cap \mathbb{T}$. In this paper, we fix $T \in \mathbb{T}$. The forward jump operator $\sigma$ is defined by 


$$
\sigma(t)=\inf \{s \in \mathbb{T}: s>t\}
$$

supplemented by inf $\varnothing:=$ sup $\mathbb{T}$, where $\varnothing$ denotes the empty set. If $\sigma(t)=t$ and $t<\sup \mathbb{T}$, the point $t$ is called right-dense, while if $\sigma(t)>t$, the point $t$ is called right-scattered. For a function $f$, we denote $f^{\sigma}(t)=f(\sigma(t))$ to represent the compositions of the functions $f$ and $\sigma$. The backward jump operator $\rho$ is defined by

$$
\rho(t)=\sup \{s \in \mathbb{T}: s<t\},
$$

supplemented by $\sup \varnothing:=\inf \mathbb{T}$. If $\rho(t)=t$ and $t>\inf \mathbb{T}$, the point $t$ is called left-dense, while if $\rho(t)<t$, the point $t$ is called left-scattered. Moreover, a point is called isolated if it is both left-scattered and right-scattered. The definition of the graininess function $\mu$ is as follows:

$$
\mu(t)=\sigma(t)-t .
$$

We now present some basic concepts and properties about time scales (see $[6,7]$ ).

Definition 1. Let $f$ be a function on $\mathbb{T} ; f$ is called a rightdense continuous function if $f$ is continuous at every rightdense point and has finite left-sided limits at every left-dense point. For an $n \times n$ matrix-valued function $A$, we say that $A$ is right-dense continuous if each entry of $A$ is right-dense continuous.

Definition 2. A function $f: \mathbb{T} \longrightarrow \mathbb{R}$ is called continuous at the point $t_{0} \in \mathbb{T}$ if $f\left(t_{n}\right) \longrightarrow f\left(t_{0}\right)$ for any sequence $t_{n} \in \mathbb{T}$ such that $t_{n} \longrightarrow t_{0}$ as $n \longrightarrow \infty$.

Define the set $\mathbb{T}^{\kappa}$ as

$$
\mathbb{T}^{\kappa}= \begin{cases}\frac{\mathbb{T}}{(\rho(\sup \mathbb{T}), \sup \mathbb{}]}, & \text { if } \sup \mathbb{}<\infty, \\ \mathbb{T}, & \text { if } \sup \mathbb{T}=\infty .\end{cases}
$$

Definition 3. Let $f: \mathbb{T} \longrightarrow \mathbb{R}$ be a function and $t \in \mathbb{T}^{\kappa}$; if for all $\varepsilon>0$, there exists a neighborhood $U$ of $t$ such that

$$
\begin{array}{r}
\left|f(\sigma(t))-f(s)-f^{\Delta}(t)(\sigma(t)-s)\right| \leq \varepsilon|\sigma(t)-s| \\
\text { for all } s \in U .
\end{array}
$$

We call $f^{\Delta}(t)$ the $\Delta$-derivative of $f$ at $t$.

If the functions $f$ and $g$ are differentiable at $t$, then the product $f g$ is also differentiable at $t$, and the product rule is given by

$$
(f g)^{\Delta}(t)=f^{\Delta}(t) g(t)+f^{\sigma}(t) g^{\Delta}(t)
$$

Proposition 1. If a function $f$ is right-dense continuous, then $f$ has antiderivative $F$.

Definition 4. A function $p$ is said to be regressive if $1+$ $\mu(t) p(t) \neq 0$ for all $t \in \mathbb{T}$. The set of all regressive and rightdense continuous functions $p: \mathbb{T} \longrightarrow \mathbb{R}$ is denoted by $\mathscr{R}$. We define $\mathscr{R}^{+}=\{p \in \mathscr{R}: 1+\mu(t) p(t)>0$ for all $t \in \mathbb{T}\}$.
For an $n \times n$ matrix-valued function $A$, if $I+\mu(t) A(t)$ is invertible for all $t \in \mathbb{T}$, we say that $A$ is regressive. Similar to the scalar case, the regressive and right-dense continuous matrix-valued function $A$ is denoted by $A \in \mathscr{R}$.

Notations: the following notations will be used:

$M^{\prime}$ : the transpose of any vector or matrix $M$

$|M|:=\sqrt{\sum_{i, j} m_{i j}^{2}}$ for any matrix or vector $M=\left(m_{i j}\right)$

$\langle X, Y\rangle_{t}$ : the quadratic covariation process of $X$ and $Y$

$S^{n}$ : the space of all $n \times n$ symmetric matrices

$S_{+}^{n}$ : the space of all $n \times n$ positive semidefinite symmetric matrices

$L^{\infty}\left([0, T]_{\mathbb{T}} ; \mathbb{R}\right)$ : the space of bounded, $\Delta$-Lebesgueintegrable, and $\mathbb{R}$-valued functions on $[0, T]_{\mathbb{T}}$

$C_{r d}\left([0, T]_{\mathbb{T}} ; \mathbb{R}\right)$ : the space of $\mathbb{R}$-valued right-dense continuous functions on $[0, T]_{\mathbb{T}}$

$C\left([0, T]_{\mathbb{T}} ; \mathbb{R}\right)$ : the space of $\mathbb{R}$-valued continuous functions on $[0, T]_{\mathbb{T}}$

$C^{1}\left([0, T]_{\mathbb{T}} ; \mathbb{R}\right)$ : the family of all $\mathbb{R}$-valued continuous functions $f(t)$ defined on $[0, T]_{\mathbb{T}}$ such that they are $\Delta$-differentiable in $t$

We now introduce two known results, which will be used in the following.

Lemma 1 (see [6]). Suppose $y \in C_{r d}\left([0, T]_{\mathbb{T}} ; \mathbb{R}\right)$ and $\alpha \in \mathscr{R}^{+}$. Then,

$$
y(t) \leq \int_{t}^{T} \alpha y^{\sigma}(s) \Delta s+y(T)
$$

implies

$$
y(t) \leq y(T) e_{\alpha}(T, t)
$$

Lemma 2 (Schur's lemma; see [25]). Let $U$ and $V$ be symmetric matrices and $Y$ be given with appropriate dimensions. The following conditions are equivalent:

(i) $U-Y V^{-1} Y^{\prime} \geq 0, V>0$,

(ii) $\left(\begin{array}{cc}U & Y \\ Y^{\prime} & V\end{array}\right) \geq 0, V>0$.

In this paper, we adopt the stochastic integral defined by Bohner et al. [10]. Let $\left(\Omega, \mathscr{F},\left\{\mathscr{F}_{t}\right\}_{t \in[0, T]_{\mathbb{T}}}, \mathbb{P}\right)$ be a complete probability space with an increasing and continuous filtration $\left\{\mathscr{F}_{t}\right\}_{t \in[0, T]_{\mathbb{T}}}$. We define that $L_{\mathscr{F}}^{2}\left([0, T]_{\mathbb{T}} ; \mathbb{R}\right)$ is the set of all $\mathscr{F}_{t}$-adapted, $\mathbb{R}$-valued measurable processes $X(t)$ such that $E\left[\int_{0}^{T}|X(t)|^{2} \Delta t\right]<\infty$.

A Brownian motion indexed by time scale $\mathbb{T}$ was defined by Grow and Sanyal [9]. Although the Brownian motion on time scales is very similar to that on continuous time, there are also some differences between them. For example, the quadratic variation of a Brownian motion on time scales (see [26]) is an increasing process yet, but it is not deterministic. In fact, $\langle W\rangle_{t}=\lambda\left([0, t]_{\mathbb{T}}\right)+\sum_{s \leq t}\left(W_{s}^{\sigma}-W_{s}\right)^{2}$, where $\lambda$ is the Lebesgue measure. 
Similar to Definition 4, we give the definition of stochastic regressive.

Definition 5 (see [27]). A function $q$ is said to be stochastic regressive if $1+q(t)(W(\sigma(t))-W(t)) \neq 0$ almost everywhere for all $t \in \mathbb{T}$, denoted by $q \in \mathscr{R}_{W}$. For an $n \times n$ matrixvalued function $B$, if $I+B(t)(W(\sigma(t))-W(t))$ is invertible almost everywhere for all $t \in \mathbb{T}$, we say that $B$ is stochastic regressive, denoted by $B \in \mathscr{R}_{W}$.

Now, we give the definition of the stochastic $\Delta$-integral and its properties.

Definition 6 (see [10]). The random process $X(t)$ is stochastic $\Delta$-integrable on $[0, T]_{\mathbb{T}}$ if the corresponding $\tilde{X}(t)$ is integrable. Furthermore, we define the integral value of $X(t)$ as

$$
\int_{0}^{T} X(t) \Delta W(t):=\int_{0}^{T} \tilde{X}(t) \mathrm{d} W(t)
$$

where

$$
\tilde{X}(t)=X\left(\sup [0, t]_{\mathbb{T}}\right) \quad \text { for all } t \in[0, T],
$$

and the Brownian motion on the right side of (9) is indexed by continuous time.

We also have the following properties.

Let $X(t), Y(t) \in L_{\mathscr{F}}^{2}\left([0, T]_{\mathbb{T}} ; \mathbb{R}\right)$ and $\alpha, \beta \in \mathbb{R}$. Then,

(i) $\begin{aligned} & \int_{0}^{T}(\alpha X(t)+\beta Y(t)) \Delta W(t)=\alpha \int_{0}^{T} X(t) \Delta W(t)+\beta \\ & \int_{0}^{T} Y(t) \Delta W(t),\end{aligned}$

(ii) $E\left[\int_{0}^{T} X(t) \Delta W(t)\right]=0$,

(iii) $E\left[\left|\int_{0}^{T} X(t) \Delta W(t)\right|^{2}\right]=E\left[\int_{0}^{T}|X(t)|^{2} \Delta\langle W\rangle_{t}\right]=E$ $\left[\int_{0}^{T}|X(t)|^{2} \Delta t\right]$ where the integral $X$ with respect to the quadratic variation of Brownian motion $\langle W\rangle_{t}$ is defined by the Stieltjes integral as $\int_{0}^{T} X_{t}(\omega) \Delta\langle W\rangle_{t}(\omega)$.

In addition, we have the following result about the stochastic $\Delta$-differential equation which is easy to be proved.

Lemma 3. Let $\Phi(\cdot)$ be the solution of the following stochastic $\Delta$-differential equation on $\left(\Omega, \mathscr{F},\left\{\mathscr{F}_{t}\right\}_{t \in[0, T]_{\mathbb{T}}}, \mathbb{P}\right)$,

$$
\left\{\begin{array}{l}
\Delta \Phi(t)=A(t) \Phi(t) \Delta t+C(t) \Phi(t) \Delta W(t), \quad t \in[0, T]_{\mathbb{T}} \\
\Phi(0)=I
\end{array}\right.
$$

$A \in \mathscr{R}$, and $(I+\mu A)^{-1} C \in \mathscr{R}_{W}$; then, $\Phi^{-1}(\cdot)$ exists.

Proof. For the continuous closed interval $\left[t_{1}, t_{2}\right]$, we introduce the following equation:

$$
\left\{\begin{array}{l}
\Delta \Psi=\Psi\left(-A+C^{2}\right) \Delta t-\Psi C \Delta W \\
\Psi\left(t_{1}\right)=I
\end{array}\right.
$$

and then $\Delta \Psi \Phi=0$ and $\Psi(t) \Phi(t)=\Phi\left(t_{1}\right)$ for all $t \in\left[t_{1}, t_{2}\right]$. If $\Phi^{-1}\left(t_{1}\right)$ exists, we can obtain that $\Phi^{-1}(t)$ exists for all $t \in\left[t_{1}, t_{2}\right]$. For a right-scattered point $t$, we have

$$
\begin{aligned}
\Phi(\sigma(t))= & {[I+\mu(t) A(t)] } \\
& \cdot\left[I+(I+\mu(t) A(t))^{-1} C(t)(W(\sigma(t))-W(t)) \Phi(t)\right] .
\end{aligned}
$$

If $\Phi^{-1}(t)$ exists, then $\Phi^{-1}(\sigma(t))$ exists. So, $\Phi^{-1}(t)$ exists due to $\Phi(0)=I$.

Finally, we introduce our $S \Delta L Q$ problem. Consider the following stochastic linear quadratic control problem:

$$
\operatorname{minimize} J(u(\cdot))=E\left\{\int_{0}^{T}\left[X^{\prime}(t) Q(t) X(t)+2 u^{\prime}(t) L(t) x(t)+u^{\prime}(t) R(t) u(t)\right] \Delta t+X^{\prime}(T) H X(T)\right\} \text {, }
$$

where the control $u \in L_{\mathscr{F}}^{2}\left([0, T]_{\mathbb{T}} ; R^{m}\right)$ and $X$ satisfies the linear $\Delta$-differential equation:

$$
\left\{\begin{array}{l}
\Delta X(t)=(A(t) X(t)+B(t) u(t)+f(t)) \Delta t+\sum_{j=1}^{d} D_{j}(t) u(t) \Delta W^{j}(t), \quad t \in[0, T]_{\mathbb{V}}, \\
X(0)=x_{0} \in R^{n} .
\end{array}\right.
$$

Here, $W=\left(W^{1}, \ldots, W^{d}\right)$ is a $d$-dimensional standard Brownian motion on time scales. And the associated coefficients satisfy $A \in L^{\infty}\left([0, T]_{\mathbb{T}: \mathbb{R}}^{n \times n}\right), B, D_{j}, L^{\prime} \in L^{\infty} \quad\left([0, T]_{\mathbb{T}: \mathbb{R}}^{n \times m}\right)$, $f \in L^{\infty}\left([0, T]_{\mathbb{T}} ; \mathbb{R}\right), \quad Q \in L^{\infty}\left([0, T]_{\mathbb{T}} ; S^{n}\right), R \in L^{\infty}\left([0, T]_{\mathbb{T}} ;\right.$ $\left.S^{m}\right), H \in S^{n}$. Obviously, equation (15) admits a unique solution under this condition (see Bohner et al. [10]).

\section{Main Results and the Riccati Equations}

By some simple calculations, it is not hard for us to obtain the following product rule for stochastic processes on time scales, which is very similar to that of Du and Dieu [8]. 
Lemma 4. For any two n-dimensional stochastic processes $X_{1}$ and $X_{2}$ with

$$
\left\{\begin{array}{l}
\Delta X_{i}(t)=b_{i}\left(t, X_{i}(t)\right) \Delta t+\sigma_{i}\left(t, X_{i}(t)\right) \Delta W(t), \quad t \in \mathbb{T}, \\
X_{i}(0)=\xi_{i},
\end{array}\right.
$$

where $b_{i}, \sigma_{i}: \mathbb{T} \times \mathbb{R}^{n} \longrightarrow \mathbb{R}^{n}$, we have

$$
\begin{aligned}
\Delta X_{1}^{\prime}(t) X_{2}(t)= & X_{1}^{\prime}(t) \Delta X_{2}(t)+\left(\Delta X_{1}^{\prime}(t)\right) X_{2}(t) \\
& +\Delta\left\langle X_{1}, X_{2}\right\rangle_{t},
\end{aligned}
$$

where

$$
\begin{aligned}
\Delta\left\langle X_{1}, X_{2}\right\rangle_{t}= & \mu(t) b_{1}^{\prime} b_{2} \Delta t+\sigma_{1}^{\prime} \sigma_{2} \Delta\langle W\rangle_{t} \\
& +\mu(t)\left(b_{1}^{\prime} \sigma_{2}+b_{2}^{\prime} \sigma_{1}\right) \Delta W(t) .
\end{aligned}
$$

Remark 1. We can also obtain another form of the above product rule as follows:

$$
\begin{aligned}
\Delta X_{1}^{\prime}(t) X_{2}(t)= & X_{1}^{\prime}(t) \Delta X_{2}(t)+\left(\Delta X_{1}^{\prime}(t)\right) X_{2}(t) \\
& +\Delta X_{1}^{\prime}(t) \Delta X_{2}(t),
\end{aligned}
$$

where $\quad \Delta t \Delta t=\mu(t) \Delta t, \quad \Delta t \Delta W=\Delta W \Delta t=\mu(t) \Delta W, \quad$ and $\Delta W \Delta W=\Delta\langle W\rangle_{t}$. When $\mathbb{T}=\mathbb{R}$, it is consistent with It $\widehat{o}$ 's formulas.

Remark 2. As mentioned before, since the quadratic variation of a process depends on not only the process itself but also the structure of the time scale, the quadratic variation of a process becomes a little more complicated than the classical one. For example, the quadratic variation of a deterministic continuous process is no longer zero. Therefore, we can have different forms of the product rule on time scales. For example, product rule (6) is equivalent to

$$
(f g)^{\Delta}(t)=f^{\Delta}(t) g(t)+f(t) g^{\Delta}(t)+\mu(t) f^{\Delta}(t) g^{\Delta}(t) .
$$

Now, we introduce the following $R \Delta E$ for our problem:

$$
\left\{\begin{array}{l}
-P^{\Delta}(t)=Q(t)+A^{\prime}(t) P^{\sigma}(t)+P^{\sigma}(t) A(t)+\mu(t) A^{\prime}(t) P^{\sigma}(t) A(t)-\left[\left(I+\mu(t) A^{\prime}(t)\right) P^{\sigma}(t) B(t)+L^{\prime}(t)\right] K^{-1}(t) \\
\quad \times\left[B^{\prime}(t) P^{\sigma}(t)(I+\mu(t) A(t))+L(t)\right] \\
K(t)=R(t)+\mu(t) B^{\prime}(t) P^{\sigma}(t) B(t)+\sum_{j=1}^{d} D_{j}^{\prime}(t) P^{\sigma}(t) D_{j}(t)>0, \quad t \in[0, T]_{\mathbb{T}} \\
P(t)=H
\end{array}\right.
$$

along with an equation on time scales:

$$
\left\{\begin{array}{l}
\Delta g(t)=\left(\Phi(t) P^{\sigma}(t) f(t)+\Psi(t) g^{\sigma}(t)\right) \Delta t, \quad t \in[0, T]_{\mathbb{T}}, \\
g(T)=0
\end{array}\right.
$$

where 0 is the zero vector and

$$
\begin{aligned}
\Phi(t)= & \mu(t)\left[\left(I+\mu(t) A^{\prime}(t)\right) P^{\sigma}(t) B(t)+L^{\prime}(t)\right] K^{-1} \\
& \cdot(t) B^{\prime}(t)-\left(\left(I+\mu(t) A^{\prime}(t)\right),\right. \\
\Psi(t)= & {\left[\left(I+\mu(t) A^{\prime}(t)\right) P^{\sigma}(t) B(t)+L^{\prime}(t)\right] K^{-1} } \\
& \cdot(t) B^{\prime}(t)-A^{\prime}(t) .
\end{aligned}
$$

Clearly, equation (22) has a unique solution since it is a linear equation (see Bohner and Peterson [7]). $R \Delta E$ (21) is very similar to the classical Riccati equation in continuous and discrete time (see $[22,28]$ ). The only difference is that $\mathrm{R} \Delta \mathrm{E}$ includes graininess function $\mu$. When $\mathbb{T}=\mathbb{R}^{+}$or $\mathbb{T}=\mathbb{Z}^{+},(21)$ can degenerate into the classical ones.

A matrix-valued function $P \in C^{1}\left([0, T]_{\pi} ; S^{n}\right)$ is called a solution to $R \Delta E$ if it satisfies (21). A function $g \in C^{1}\left([0, T]_{\mathbb{T}} ; \mathbb{R}^{n}\right)$ is a solution to (22) if it satisfies the $\Delta$-differential equation. Here, we use the square completion technique to show that the solutions of equations (21) and (22) can give a state-feedback optimal control.
Theorem 1. Suppose that $P \in C^{1}\left([0, T]_{\mathbb{T}} ; S^{n}\right)$ and $g \in C^{1}$ $\left([0, T]_{\mathbb{T}} ; \mathbb{R}^{n}\right)$ are solutions of $(21)$ and $(22)$, respectively; then, $S \triangle L Q$ problem (14) and (15) have an optimal control $\bar{u}$ :

$$
\begin{aligned}
\bar{u}(t)= & -K^{-1}(t)\left\{B^{\prime}(t) P^{\sigma}(t)[(I+\mu(t) A(t))+L(t)] X(t)\right. \\
& \left.+\mu(t) B^{\prime}(t) P^{\sigma}(t) f(t)+B^{\prime}(t) g^{\sigma}(t)\right\},
\end{aligned}
$$

and the optimal cost functional $\bar{J}$ is

$$
\begin{aligned}
\bar{J}= & x_{0}^{\prime} P(0) x_{0}+2 x_{0}^{\prime} g(0)-\int_{0}^{T}\left(g^{\sigma}(t)\right)^{\prime} B(t) K^{-1} \\
& \cdot(t) B^{\prime}(t) g^{\sigma}(t) \Delta t+\int_{0}^{T} \mu(t) f^{\prime}(t) \\
& \cdot\left[I-\mu(t) P^{\sigma}(t) B(t) K^{-1}(t) B^{\prime}(t)\right] P^{\sigma}(t) f(t) \Delta t \\
& +2 \int_{0}^{T} f^{\prime}(t)\left[I-\mu(t) P^{\sigma}(t) B(t) K^{-1}(t) B^{\prime}(t)\right] g^{\sigma}(t) \Delta t .
\end{aligned}
$$

Proof. Applying the product rule to $X(t)^{\prime} P(t) X(t)$ and $X^{\prime}(t) g(t)$, we can obtain that 


$$
\begin{aligned}
\Delta X^{\prime}(t) P(t) X(t)= & \left\{X ^ { \prime } ( t ) \left[\left(\left(I+\mu(t) A^{\prime}(t)\right) P^{\sigma}(t) B(t)+L^{\prime}(t)\right) K^{-1}(t) \times\left(B^{\prime}(t) P^{\sigma}(t)((I+\mu(t) A(t))+L(t))-Q(t)\right] X(t)\right.\right. \\
& +\mu(t) u^{\prime}(t) B^{\prime}(t) P^{\sigma}(t) B(t) u(t)+2 u^{\prime}(t) B^{\prime}(t) P^{\sigma}(t) \times[(I+\mu(t) A(t)) X(t)+\mu(t) f(t)] \\
& \left.+2 X^{\prime}(t)\left(I+\mu(t) A^{\prime}(t)\right) P^{\sigma}(t) f(t)+\mu(t) f^{\prime}(t) P^{\sigma}(t) f(t)\right\} \Delta t \\
& +\sum_{i=1}^{d} \sum_{j=1}^{d} u^{\prime}(t) D_{i}^{\prime}(t) P^{\sigma}(t) D_{j}(t) u(t) \Delta\left\langle W^{i}, W^{j}\right\rangle_{t}+2[X(t)+\mu(t)(A(t) X(t)+B(t) u(t)+f(t))]^{\prime} \\
& \times P^{\sigma}(t) \sum_{j=1}^{d} D_{j}(t) u(t) \Delta W^{j}(t), \quad t \in[0, T]_{\mathbb{T}}, \\
\Delta X^{\prime}(t) g(t)=\{ & \left.X^{\prime}(t)\left(\Phi(t) P^{\sigma}(t) f(t)+\Psi(t) g^{\sigma}(t)\right)+(A(t) X(t)+B(t) u(t)+f(t))^{\prime} g^{\sigma}(t)\right\} \Delta t \\
& +\sum_{j=1}^{d} u^{\prime}(t) D_{j}^{\prime}(t) g^{\sigma}(t) \Delta W^{j}(t), \quad t \in[0, T]_{\mathbb{V}} \cdot
\end{aligned}
$$

Integrating from 0 to $T$, taking expectation on both sides of the above two equations, and then adding them into the cost functional (14) yield

$$
\begin{aligned}
J(u(\cdot))= & x_{0}^{\prime} P(0) x_{0}+2 x_{0}^{\prime} g(0)-\int_{0}^{T}\left(g^{\sigma}(t)\right) B(t) K^{-1}(t) B^{\prime}(t) g^{\sigma}(t) \Delta t \\
& +\int_{0}^{T} \mu(t) f^{\prime}(t)\left(I-\mu(t) P^{\sigma}(t) B(t) K^{-1}(t) B^{\prime}(t)\right) P^{\sigma}(t) f(t) \Delta t \\
& +2 \int_{0}^{T} f^{\prime}(t)\left(I-\mu(t) P^{\sigma}(t) B(t) K^{-1}(t) B^{\prime}(t)\right) g^{\sigma}(t) \Delta t \\
& +E\left\{\int_{0}^{T}\left[u(t)+K^{-1}(t)\left[B^{\prime}(t) P^{\sigma}(t)((I+\mu(t) A(t))+L(t)) X(t)+\mu(t) B^{\prime}(t) P^{\sigma}(t) f(t)+B^{\prime}(t) g^{\sigma}(t)\right]^{\prime}\right] K(t)\right. \\
& \left.\times\left[u(t)+K^{-1}(t) B^{\prime}(t) P^{\sigma}(t)((I+\mu(t) A(t))+L(t)) X(t)+\mu(t) B^{\prime}(t) P^{\sigma}(t) f(t)+B^{\prime}(t) g^{\sigma}(t)\right] \Delta t\right\} .
\end{aligned}
$$

Since $K>0$, it follows that the optimal control is the form of (25), and the minimum value of the cost functional is (26).

Remark 3. Obviously, if $f \equiv 0$, we just need to think about $R \Delta E$ (21). And we can see from the above proof that the uniqueness of the solutions to (21) and (22) is not necessary for the establishment of (25) and (26).

It is worth pointing out that the state feedback matrix relies on not only the solution of $\mathrm{R} \Delta \mathrm{E}$ but also the graininess function $\mu$. In addition to this, (25) and (26) are very similar to the classical cases.

Now, we discuss the existence and uniqueness of the solution to $R \Delta E$. We will apply a technique given in [29].

Theorem 2. Assume that $P \in C^{1}\left([0, T]_{\mathbb{T}} ; S^{n}\right)$ is a solution to $R \Delta E$ (21); then, $P$ is unique.

Proof. Suppose that $\widetilde{P} \in C^{1}\left([0, T]_{\mathbb{T}} ; S^{n}\right)$ is another solution of $R \Delta E$ (21). We consider a new $\mathrm{S} \Delta \mathrm{LQ}$ problem as follows:

$$
\begin{aligned}
& \operatorname{minimize} J(s, y ; u(\cdot))=E\left[\int_{s}^{T}\left(X^{\prime}(t) Q(t) X(t)+2 u^{\prime}(t) L(t) X(t)+u^{\prime}(t) R(t) u(t)\right) \Delta t+X^{\prime}(T) \mathrm{HX}(T)\right], \\
& \text { s.t. }\left\{\Delta X(t)=(A(t) X(t)+B(t) u(t)) \Delta t+\sum_{j=1}^{d} D_{j}(t) u(t) \Delta W^{j}(t), \quad t \in[s, T]_{\mathbb{T}}, X(s)=y .\right.
\end{aligned}
$$


According to the proof of Theorem 1, $y^{\prime} P(s) y$ and $y^{\prime} \widetilde{P}(s) y$ are both minimum values of the cost functional $J(s, y ; u(\cdot))$. Therefore, we obtain that $P(s)=\widetilde{P}(s)$. Due to the arbitrariness of $(s, y)$, it follows that $P=\widetilde{P}$.

For the existence of the solution to $R \Delta E$ (21), we first consider a special case:

$$
\text { (H1): } R>0, H \geq 0 \text {, and } Q-L^{\prime} R^{-1} L \geq 0 .
$$

In this case, we study a linear equation required in the following.

Proposition 2. The linear matrix-valued $\Delta$-differential equation

$$
\left\{\begin{array}{l}
-P^{\Delta}(t)=A^{\prime}(t) P^{\sigma}(t)+P^{\sigma}(t) A(t)+\mu(t) A^{\prime}(t) P^{\sigma}(t) A(t)+\sum_{j=1}^{d} C_{j}^{\prime}(t) P^{\sigma}(t) C_{j}(t)+Q(t), \quad t \in[0, T]_{\mathbb{T}}, \\
P(T)=H,
\end{array}\right.
$$

where $A, C_{j} \in L^{\infty}\left([0, T]_{\mathbb{\pi}: \mathbb{R}}^{n \times n}\right), \quad Q \in L^{\infty}\left([0, T]_{\mathbb{T}} ; S^{n}\right)$, and $\quad$ Proof. Let $P_{0}(t) \equiv H$ and $H \in S^{n}$, has a unique solution $P \in C^{1}\left([0, T]_{\mathbb{T}} ; S^{n}\right)$.

$$
P_{k+1}(t)=H+\int_{t}^{T}\left(A^{\prime}(s) P_{k}^{\sigma}(s)+P_{k}^{\sigma}(s) A(s)+\mu(s) A^{\prime}(s) P_{k}^{\sigma}(s) A(s)+\sum_{j=1}^{d} C_{j}^{\prime}(s) P_{k}^{\sigma}(s) C_{j}(s)+Q(s)\right) \Delta s, \quad t \in[0, T]_{\mathbb{T}} .
$$

Then,

$$
\begin{aligned}
P_{k+1}(t)-P_{k}(t)= & \int_{t}^{T}\left\{A^{\prime}(s)\left(P_{k}^{\sigma}(s)-P_{k-1}^{\sigma}(s)\right)+\left(P_{k}^{\sigma}(s)-P_{k-1}^{\sigma}(s)\right) A(s)+\mu(s) A^{\prime}(s)\left(P_{k}^{\sigma}(s)-P_{k-1}^{\sigma}(s)\right) A(s)\right. \\
& \left.+\sum_{j=1}^{d} C_{j}^{\prime}(s)\left(P_{k}^{\sigma}(s)-P_{k-1}^{\sigma}(s)\right) C_{j}(s)\right\} \Delta s, \quad t \in[0, T]_{\mathbb{T}}, \\
P_{1}(t)-P_{0}(t)= & \int_{t}^{T}\left\{A^{\prime}(s) H(s)+H(s) A(s)+\mu(s) A^{\prime}(s) H(s) A(s)+\sum_{j=1}^{d} C_{j}^{\prime}(s) H(s) C_{j}(s)+Q(s)\right\} \Delta s, \quad t \in[0, T]_{\mathbb{T}} .
\end{aligned}
$$

Because of the boundedness of $A, C_{j}, H$, and $Q$, there exists a constant $M>0$ such that

$$
\begin{aligned}
& \left|P_{k+1}(t)-P_{k}(t)\right| \leq M \int_{t}^{T}\left|P_{k}^{\sigma}(s)-P_{k-1}^{\sigma}(s)\right| \Delta s, \quad t \in[0, T]_{\mathbb{T}}, \\
& \left|P_{1}(t)-P_{0}(t)\right| \leq M(T-t) .
\end{aligned}
$$

Consequently, we have that

$$
\left|P_{k+1}(t)-P_{k}(t)\right| \leq M^{k+1} h_{k+1}(T, t), \quad t \in[0, T]_{\mathbb{T}},
$$

where $h_{k}$ is the generalized monomials defined in [30]. Thus, $\sum_{k}\left|P_{k+1}(t)-P_{k}(t)\right|$ is uniformly convergent. Moreover, $P_{k}$ uniformly converges to $P, P$ is a solution of (32), and we get the existence of a solution to (32). If $\widetilde{P}$ is another solution of (32), then 


$$
\left\{\begin{array}{l}
-\widehat{P}^{\Delta}(t)=A^{\prime}(t) \widehat{P}^{\sigma}(t)+\widehat{P}^{\sigma}(t) A(t)+\mu(t) A^{\prime}(t) \widehat{P}^{\sigma}(t) A(t)+\sum_{j=1}^{d} C_{j}^{\prime}(t) \widehat{P}^{\sigma}(t) C_{j}(t), \quad t \in[0, T]_{\mathbb{T}} \\
\widehat{P}(T)=0
\end{array}\right.
$$

where $\widehat{P}=P-\widetilde{P}$. Based on some properties of matrix norms, there is a constant $\alpha$ such that

$$
|\widehat{P}(t)| \leq \int_{t}^{T} \alpha\left|\widehat{P}^{\sigma}(s)\right| \Delta s+|\widehat{P}(T)|
$$

By Lemma 1 , it is easy to obtain $\widehat{P}=0$. This implies the uniqueness of a solution to (32).

For linear equation (32), we also have the following property.
Proposition 3. Let $Q$ and $H$ be positive semidefinite symmetric matrices; then, the solution $P$ of (32) is also a positive semidefinite symmetric matrix.

Proof. For the continuous closed interval $\left[t_{1}, t_{2}\right] \in[0, T]_{\mathbb{T}}$ and $t_{1}, t_{2} \in[0, T]_{\mathbb{T}}$, by (11) and (32) and applying product rules to $\Phi^{\prime}(t) P(t) \Phi(t)$, we get

$$
\begin{aligned}
\Delta \Phi^{\prime}(t) P(t) \Phi(t)= & -\Phi^{\prime}(t)\left(\sum_{j=1}^{d} C_{j}^{\prime}(t) P^{\sigma}(t) C_{j}(t)+Q(t)\right) \Phi(t) \Delta+\sum_{j=1}^{d} C_{j}^{\prime}(t) P^{\sigma}(t) C_{j}(t) \Phi(t) \Delta\langle W\rangle_{t} t \\
& +\sum_{j=1}^{d} \Phi^{\prime}(t)\left\{C_{j}^{\prime}(t) P^{\sigma}(t)+P^{\sigma}(t) C_{j}(t)+\mu(t) A^{\prime}(t) P^{\sigma}(t) C_{j}(t)+\mu(t) C_{j}^{\prime}(t) P^{\sigma}(t) A(t)\right\} \\
& . \Phi(t) \Delta W(t), \quad t \in\left[t_{1}, t_{2}\right]_{\mathbb{T}} .
\end{aligned}
$$

Therefore,

$$
P(t)=E\left[\left(\Phi^{\prime}(t)\right)^{-1} \Phi^{\prime}\left(t_{2}\right) P\left(t_{2}\right) \Phi\left(t_{2}\right)\left(\Phi^{\prime}(t)\right)^{-1}+\int_{t}^{T}\left(\Phi^{\prime}(t)\right)^{-1} \Phi^{\prime}(s) Q(s) \Phi(s)\left(\Phi^{\prime}(t)\right)^{-1} \Delta s\right], \quad t \in\left[t_{1}, t_{2}\right]_{\mathbb{T}}
$$

If $P\left(t_{2}\right) \geq 0$, we have $P(t) \geq 0$ for all $t \in\left[t_{1}, t_{2}\right]_{\mathbb{T}}$. For right-scattered point $t \in[0, T]_{\mathbb{T}}$, we have

$$
\begin{aligned}
P(t)= & \left(I+\mu(t) A^{\prime}(t)\right) P^{\sigma}(t)(I+\mu(t) A(t)) \\
& +\mu(t) \sum_{j=1}^{d} C_{j}^{\prime}(t) P^{\sigma}(t) C_{j}(t)+\mu(t) Q(t) .
\end{aligned}
$$

If $P^{\sigma}(t) \geq 0$, then $P(t) \geq 0$. From the above, since $P(T)=H \geq 0$, we have $P(t) \geq 0$ for all $t \in[0, T]_{\mathbb{T}}$.

Furthermore, we have an equivalent form of $\mathrm{R} \Delta \mathrm{E}$ (21) under assumption (H1).

Lemma 5. Assume (H1) holds; $R \Delta E$ (21) is equivalent to the following equation:

$$
\left\{\begin{array}{l}
-P^{\Delta}(t)=\widehat{A}^{\prime}(t) P^{\sigma}(t)+P^{\sigma}(t) \widehat{A}(t)+\mu(t) \widehat{A}^{\prime}(t) P^{\sigma}(t) \widehat{A}(t)+\sum_{j=1}^{d} \widehat{C}_{j}^{\prime}(t) P^{\sigma}(t) \widehat{C}_{j}(t)+\widehat{Q}(t), \quad t \in[0, T]_{\mathbb{T}}, \\
P(T)=H
\end{array}\right.
$$

where

$$
\left\{\begin{array}{l}
\widehat{A}(t)=A(t)-B(t) \Psi(t), \quad \widehat{C}_{j}(t)=-D_{j}(t) \Psi(t), \\
\widehat{Q}(t)=\left(\Psi(t)-R^{-1(t)} L(t)\right)^{\prime} R(t)\left(\Psi(t)-R^{-1}(t) L(t)\right)+Q(t)-L^{\prime}(t) R^{-1}(t) L(t), \\
\Psi(t)=\left[R(t)+\mu(t) B^{\prime}(t) P^{\sigma}(t) B(t)+\sum_{j=1}^{d} D_{j}(t) P^{\sigma}(t) D_{j}(t)\right]^{-1} \times\left[B^{\prime}(t) P^{\sigma}(t)(I+\mu(t) A(t))+L(t)\right] \quad t \in[0, T]_{\mathbb{T}} .
\end{array}\right.
$$


Proof. By (45), we have

$$
\begin{aligned}
\Psi^{\prime}(t)\left(R(t)+\mu(t) B^{\prime}(t) P^{\sigma}(t) B(t)+\sum_{j=1}^{d} D_{j}(t) P^{\sigma}(t) D_{j}(t)\right) \Psi(t) & \\
= & \Psi^{\prime}(t)\left(B^{\prime}(t) P^{\sigma}(t)(I+\mu(t) A(t))+L(t)\right) \\
= & \left(\left(I+\mu(t) A^{\prime}(t)\right) P^{\sigma}(t) B(t)+L^{\prime}(t)\right) \Psi(t), \\
-P^{\Delta}(t)= & A^{\prime}(t) P^{\sigma}(t)+P^{\sigma}(t) A(t)+\mu(t) A^{\prime}(t) P^{\sigma}(t) A(t)+Q(t)-\left(\left(I+\mu(t) A^{\prime}(t)\right) P^{\sigma}(t) B(t)+L(t)\right) K^{-1} \\
& \cdot(t)\left(B^{\prime}(t) P^{\sigma}(t)(I+\mu(t) A(t))+L(t)\right) \\
= & \widehat{A}^{\prime}(t) P^{\sigma}(t)+P^{\sigma}(t) \widehat{A}(t)+\mu(t) \widehat{A}(t) P^{\sigma}(t) \widehat{A}(t)+Q(t)+\left[\left(I+\mu(t) A^{\prime}(t)\right) P^{\sigma}(t) B(t)+L^{\prime}(t)\right] \Psi \\
& \cdot(t)-L^{\prime}(t) \Psi(t)+\Psi^{\prime}(t)\left[B^{\prime}(t) P^{\sigma}(t)(I+\mu(t) A(t))+L(t)\right] \\
& -\Psi^{\prime}(t) L(t)-\Psi^{\prime}(t)\left[R(t)+\mu(t) B^{\prime}(t) P^{\sigma}(t) B(t)+\sum_{j=1}^{d} D_{j}(t) P^{\sigma}(t) D_{j}(t)\right] \Psi(t)-\mu(t) \Psi^{\prime}(t) B^{\prime}(t) P^{\sigma}(t) B(t) \Psi(t) \\
= & \widehat{A}^{\prime}(t) P^{\sigma}(t)+P^{\sigma}(t) \widehat{A}(t)+\mu(t) \widehat{A}(t) P^{\sigma}(t) \widehat{A}(t)+\Psi^{\prime}(t) R(t) \Psi(t)+\Psi^{\prime} \\
& \cdot(t) \sum_{j=1}^{d} D_{j}^{\prime}(t) P^{\sigma}(t) D_{j}(t) \Psi(t)-L^{\prime}(t) \Psi(t)-\Psi^{\prime}(t) L(t)+Q(t) \\
& \widehat{A}^{\prime}(t) P^{\sigma}(t)+P^{\sigma}(t) \widehat{A}(t)+\mu(t) \widehat{A}(t) P^{\sigma}(t) \widehat{A}(t)+\sum_{j=1}^{d} \widehat{C}_{j}^{\prime}(t) P^{\sigma}(t) \widehat{C}{ }_{j}(t)+\widehat{Q}(t) .
\end{aligned}
$$

The proof is completed.

Consequently, the existence of the solution to (44) is equivalent to the existence of the solution to (21) under assumption (H1).
Theorem 3. Let (H1) hold; then $R \Delta E$ (21) admits a positive semidefinite symmetric solution.

Proof. Let $P_{i+1}$ be the solution of

$$
\left\{\begin{array}{l}
-P_{i+1}^{\Delta}(t)=\widehat{A}_{i}^{\prime}(t) P_{i+1}^{\sigma}(t)+P_{i+1}^{\sigma}(t) \widehat{A}_{i}(t)+\mu(t) \widehat{A}_{i}^{\prime}(t) P_{i+1}^{\sigma}(t) \widehat{A}_{i}(t)+\sum_{j=1}^{d} \widehat{C}_{i j}^{\prime}(t) P_{i+1}^{\sigma}(t) \widehat{C}_{i j}(t)+\widehat{Q}_{i}(t), \quad t \in[0, T]_{\mathbb{T}} \\
P_{i+1}(T)=H
\end{array}\right.
$$

where

$$
\left\{\begin{array}{l}
P_{0}(t)=H \\
\widehat{A}_{i}(t)=A(t)-B(t) \Psi_{i}(t), \quad \widehat{C}_{i j}(t)=-D_{j}(t) \Psi_{i}(t), \\
\widehat{Q}_{i}(t)=\left(\Psi_{i}(t)-R^{-1}(t) L(t)\right)^{\prime} R(t)\left(\Psi_{i}(t)-R^{-1}(t) L(t)\right)+Q(t)-L^{\prime}(t) R^{-1}(t) L(t), \\
\Psi_{i}(t)=\left[R(t)+\mu(t) B^{\prime}(t) P_{i}^{\sigma}(t) B(t)+\sum_{j=1}^{d} D_{j}^{\prime}(t) P_{i}^{\sigma}(t) D_{j}(t)\right]^{-1} \times\left[B^{\prime}(t) P_{i}^{\sigma}(t)(I+\mu(t) A(t))+L(t)\right], \quad t \in[0, T]_{\mathbb{V}}
\end{array}\right.
$$


By Proposition 3, we can see that $P_{i} \geq 0$.

We will claim that $P_{i} \geq P_{i+1}$. Set $M_{i}=P_{i}-P_{i+1}$; then, $M_{i}$ satisfies

$$
\left\{\begin{array}{l}
-M_{i}^{\Delta}(t)=\widehat{A}_{i}^{\prime}(t) M_{i}^{\sigma}(t)+M_{i}^{\sigma}(t) \widehat{A}_{i}(t)+\mu(t) \widehat{A}_{i}^{\prime}(t) M_{i}^{\sigma}(t) \widehat{A}_{i}(t)+\sum_{j=1}^{d} \widehat{C}_{i j}^{\prime}(t) M_{i}^{\sigma}(t) \widehat{C}_{i j}(t)+N(t), \quad t \in[0, T]_{\mathbb{T}} \\
M_{i}(T)=0
\end{array}\right.
$$

where

$$
\begin{aligned}
N(t)= & \sum_{j=1}^{d} \widehat{C}_{i-1, j}^{\prime}(t) P_{i}^{\sigma}(t) \widehat{C}_{i-1, j}(t)-\sum_{j=1}^{d} \widehat{C}_{i j}^{\prime}(t) P_{i}^{\sigma}(t) \widehat{C}_{i j}(t)+\widehat{Q}_{i-1}(t)-\widehat{Q}_{i}(t) \\
& +\left(\widehat{A}_{i-1}^{\prime}(t)-\widehat{A}_{i}^{\prime}(t)\right) P_{i}^{\sigma}(t)+P_{i}^{\sigma}(t)\left(\widehat{A}_{i-1}(t)-\widehat{A}_{i}(t)\right)+\mu(t) \widehat{A}_{i-1}^{\prime}(t) P_{i}^{\sigma}(t) \widehat{A}_{i-1}(t)-\mu(t) \widehat{A}_{i}^{\prime}(t) P_{i}^{\sigma}(t) \widehat{A}_{i}(t) .
\end{aligned}
$$

An equivalent form of $N(t)$ can also be written as follows:

$$
N(t)=\left(\Psi_{i}(t)-\Psi_{i-1}(t)\right)^{\prime}\left[R(t)+\mu(t) B^{\prime}(t) P_{i}^{\sigma}(t) B(t)+\sum_{j=1}^{d} D_{j}^{\prime}(t) P_{i}^{\sigma}(t) D_{j}(t)\right] \times\left(\Psi_{i}(t)-\Psi_{i-1}(t)\right) \geq 0, \quad t \in[0, T]_{\mathbb{T}} .
$$

Noting that $M_{i}(T)=0$, by Proposition 3 once again, we get $M_{i} \geq 0$. Thus, $\left\{P_{i}\right\}_{i \geq 0}$ is a decreasing sequence and has a limit $P$. Clearly, $P$ is the solution to (44). Hence, $P$ is also the solution to $\mathrm{R} \Delta \mathrm{E}$ (21) by Lemma 5 .

Now, we prove the following sufficient and necessary conditions of the existence of the solution to $R \Delta E$ (21).

Theorem 4. $R \Delta E$ (21) admits a solution if and only if there exists $\widetilde{P}$ which satisfies the following conditions:

(i) $\widetilde{P}(T) \leq H$

(ii) $R(t)+\mu(t) B^{\prime}(t) \widetilde{P}^{\sigma}(t) B(t)+\sum_{j=1}^{d} D_{j}^{\prime}(t) \widetilde{P}^{\sigma}$ $(t)>0, \quad t \in[0, T]_{\mathbb{T}}$ $(t) D_{j}$ (iii) $\widetilde{P}$ satisfies the linear matrix inequality

$(L M I)$ condition: $\quad\left(\widetilde{P}^{\Delta}+A^{\prime} \widetilde{P}^{\sigma}+\widetilde{P}^{\sigma} A+\mu A^{\prime} \widetilde{P}^{\sigma} A+Q I+\right.$ $\left.\mu A^{\prime}\right) \widetilde{P}^{\sigma} B+L B^{\prime} \widetilde{P}^{\sigma}(I+\mu A)+L k+\mu B^{\prime} \widetilde{P}^{\sigma} B+\sum_{j=1}^{d} D_{j}^{\prime}$ $\left.\widetilde{P}^{\sigma} D_{j}\right) \geq 0$

Proof. If R $\triangle \mathrm{E}$ (21) has a solution $P$, then $P(T)=H$ and $R+\mu B^{\prime} P^{\sigma} B+\sum_{j=1}^{d} D_{j}^{\prime} P^{\sigma} D_{j}>0$. By Lemma 2, where $U=P^{\Delta}+A^{\prime} P^{\sigma}+P^{\sigma} A+\mu A^{\prime} P^{\sigma} A+Q, Y=\left(I+\mu A^{\prime}\right) P^{\sigma} B+$ $L$, and $V=R+\mu B^{\prime} P^{\sigma} B+\sum_{j=1}^{d} D_{j}^{\prime} P^{\sigma} D_{j}$, we have

$$
\left(\begin{array}{cc}
P^{\Delta}+A^{\prime} P^{\sigma}+P^{\sigma} A+\mu A^{\prime} P^{\sigma} A+Q & \left(I+\mu A^{\prime}\right) P^{\sigma} B+L \\
B^{\prime} P^{\sigma}(I+\mu A)+L^{\prime} & R+\mu B^{\prime} P^{\sigma} B+\sum_{j=1}^{d} D_{j}^{\prime} P^{\sigma} D_{j}
\end{array}\right) \geq 0
$$




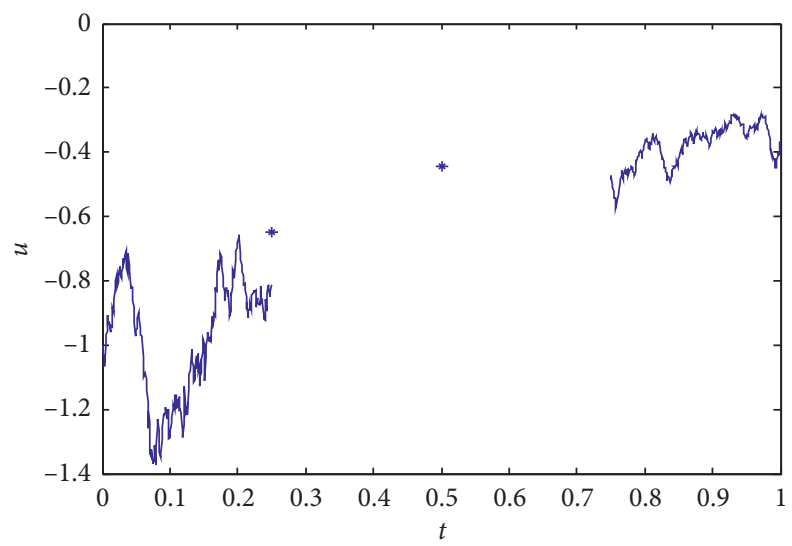

Figure 1: Optimal control of the dynamic system on time scale $\mathbb{T}=[0,1 / 4] \cup\{1 / 2\} \cup[3 / 4,1]$.

namely, $P$ satisfies conditions (i)-(iii).

Conversely, suppose there exists $\widetilde{P}$ which satisfies (i)-(iii), and define

$$
\left\{\begin{array}{l}
\widehat{Q}(t)=\widetilde{P}^{\Delta}(t)+A^{\prime}(t) \widetilde{P}^{\sigma}(t)+\widetilde{P}^{\sigma}(t) A(t)+\mu(t) A^{\prime}(t) \widetilde{P}^{\sigma}(t) A(t)+Q(t), \\
\widehat{R}(t)=R(t)+\mu(t) B^{\prime}(t) \widetilde{P}^{\sigma}(t) B(t)+\sum_{j=1}^{d} D_{j}^{\prime}(t) \widetilde{P}^{\sigma}(t) D_{j}(t) \\
\widehat{L}(t)=B^{\prime}(t) \widetilde{P}^{\sigma}(t)(I+\mu(t) A(t))+L(t), \quad t \in[0, T]_{\mathbb{T}}
\end{array}\right.
$$

Thus, $\widehat{R}>0$. Moreover, Lemma 2 yields $\widehat{Q}-\widehat{L}^{\prime} \widehat{R} \widehat{L} \geq 0$.

Now, consider a new Riccati equation:

$$
\left\{\begin{array}{l}
-Z^{\Delta}(t)=\widehat{Q}(t)+A^{\prime}(t) Z^{\sigma}(t)+Z^{\sigma}(t) A(t)+\mu(t) A^{\prime}(t) Z^{\sigma}(t) A(t)-\left[\left(I+\mu(t) A^{\prime}(t)\right) Z^{\sigma}(t) B(t)+\widehat{L}^{\prime}(t)\right] \\
\quad \times\left[\widehat{R}(t)+\mu B^{\prime}(t) Z^{\sigma}(t) B(t)+\sum_{j=1}^{d} D_{j}^{\prime}(t) Z^{\sigma}(t) D_{j}(t)\right]^{-1} \times\left[B^{\prime}(t) Z^{\sigma}(t)(I+\mu(t) A(t))+\widehat{L}(t)\right] \\
\widehat{R}(t)+\mu(t) B^{\prime}(t) Z^{\sigma}(t) B(t)+\sum_{j=1}^{d} D_{j}^{\prime}(t) Z^{\sigma}(t) D_{j}(t)>0, \quad t \in[0, T]_{\mathbb{T}} \\
Z(T)=H-\widetilde{P}(T) .
\end{array}\right.
$$

According to Theorem 3, the above equation has a solution $Z \geq 0$. Let $P=Z+\widetilde{P}$; it is obvious that $R+\mu B^{\prime} P^{\sigma} B+$ $\sum_{j=1}^{d} D_{j}^{\prime} P^{\sigma} D_{j}>0$ and $P(T)=H$. By some manipulations, we can easily verify that $P$ is a solution to $R \Delta E$ (21). This completes the proof.

Remark 4. Note that if $\mu \equiv 0$, conditions (i)-(iii) are consistent with the result of Theorem 3.3 in [29].

\section{Example}

In this section, in order to illustrate our result, we give an example. Consider a simple Mayer problem on time scale $\mathbb{T}=[0,1 / 4] \cup\{1 / 2\} \cup[3 / 4,1]$ as follows:

$$
\begin{aligned}
& \text { minimize } J=E\left[x^{2}(1)\right], \\
& \text { subject to }\left\{\begin{array}{l}
\Delta x(t)=u(t) \Delta t+u(t) \Delta W(t), \\
x(0)=1 .
\end{array}\right.
\end{aligned}
$$

The corresponding dynamic Riccati equation is

$$
\left\{\begin{array}{l}
\Delta P(t)=\frac{1}{1+\mu(t)} P^{\sigma}(t) \Delta t \\
P(1)=1 \\
(1+\mu(t)) P^{\sigma}(t)>0 .
\end{array}\right.
$$

We have $P(t)=e_{1 / 1+\mu(t)}(1, t)$. The optimal control $\bar{u}(t)=-1 / 1+\mu(t) x(t)$ (see Figure 1). 
We can see that the optimal strategy depends on the structure of time scale $\mathbb{T}=[0,1 / 4] \cup\{1 / 2\} \cup[3 / 4,1]$. Furthermore, the example implies that we should take an impulsive control when $t=1 / 4$ in the time scale setting. This interesting result is hidden in the classical continuous and discrete formulation. It reveals that we should use an impulsive control method at the right-scattered points. In addition, the impulsive control relies on the time gap.

\section{Conclusion}

The linear quadratic optimal control problems for stochastic differential equations on time scales are studied. They unify and extend the optimal control problems in continuous- and discrete-time formulations. Through the Riccati equation on time scales, we get the corresponding optimal control with the state feedback representation. The optimal control problems established in this paper offer a more practical scheme in directly tackling the issue on the mixture of continuous time and discrete time.

\section{Data Availability}

The data used to support the findings of this study are included within the article.

\section{Conflicts of Interest}

The authors declare that there are no conflicts of interest regarding the publication of this paper.

\section{Acknowledgments}

This work was supported by the National Key R\&D Program of China (Grant no. 2018YFA0703900) and the Major Project of the National Social Science Foundation of China (Grant no. 19ZDA091).

\section{References}

[1] R. E. Kalman, "Contributions to the theory of optimal control," Boletin Sociedad Matematica Mexicana, vol. 5, no. 2, pp. 102-119, 1960.

[2] M. Davis, Linear Estimation and Stochastic Control, Chapman \& Hall, London, UK, 1977.

[3] J. Yong and X. Y. Zhou, Stochastic Controls: Hamiltonian Systems and HJB Equations, Springer, New York, NY, USA, 1999.

[4] S. Hilger, "Analysis on measure chains-a unified approach to continuous and discrete calculus," Results in Mathematics, vol. 18, no. 1-2, pp. 18-56, 1990.

[5] G. S. Guseinov, "Integration on time scales," Journal of Mathematical Analysis and Applications, vol. 285, no. 1, pp. 107-127, 2003.

[6] M. Bohner and A. Peterson, Dynamic Equations on Time Scales: An Introduction with Applications, Birkhäuser, Boston, MA, USA, 2001.

[7] M. Bohner and A. Peterson, Advances in Dynamic Equations on Time Scales, Birkhäuser, Boston, MA, USA, 2002.

[8] N. H. Du and N. T. Dieu, "The first attempt on the stochastic calculus on time scale," Stochastic Analysis and Applications, vol. 29, no. 6, pp. 1057-1080, 2011.
[9] D. Grow and S. Sanyal, "Brownian motion indexed by a time scale," Stochastic Analysis and Applications, vol. 29, no. 3, pp. 457-472, 2011.

[10] M. Bohner, O. M. Stanzhytskyi, and A. O. Bratochkina, "Stochastic dynamic equations on general time scales," Electronic Journal of Differential Equations, vol. 2013, no. 57, pp. 1-15, 2013.

[11] M. Bohner, "Calculus of variations on time scales," Dynamic Systems and Applications, vol. 13, no. 3-4, pp. 339-349, 2004.

[12] F. M. Atici, D. C. Biles, and A. Lebedinsky, "An application of time scales to economics," Mathematical and Computer Modelling, vol. 43, no. 7-8, pp. 718-726, 2006.

[13] M. Bohner and G. S. Guseinov, "Double integral calculus of variations on time scales," Computers \& Mathematics with Applications, vol. 54, no. 1, pp. 45-57, 2007.

[14] Z. Zhan and W. Wei, "On existence of optimal control governed by a class of the first-order linear dynamic systems on time scales," Applied Mathematics and Computation, vol. 215, no. 6, pp. 2070-2081, 2009.

[15] Y. Peng, X. Xiang, and Y. Jiang, "Nonlinear dynamic systems and optimal control problems on time scales," ESAIM: Control, Optimisation and Calculus of Variations, vol. 17, no. 3, pp. 654-681, 2011.

[16] M. Bohner, K. Kenzhebaev, O. Lavrova, and O. Stanzhytskyi, "Pontryagin's maximum principle for dynamic systems on time scales," Journal of Difference Equations and Applications, vol. 23, no. 7, pp. 1161-1189, 2017.

[17] J. Seiffertt, S. Sanyal, and D. C. Wunsch, "Hamilton-JacobiBellman equations and approximate dynamic programming on time scales," IEEE Transactions on Systems, Man, and Cybernetics, Part B (Cybernetics), vol. 38, no. 4, pp. 918-923, 2008.

[18] M. Bohner and N. Wintz, "The linear quadratic regulator on time scales," International Journal of Difference Equations, vol. 5, no. 2, pp. 149-174, 2010.

[19] R. S. Hilscher and V. Zeidan, "Hamilton-Jacobi theory over time scales and applications to linear-quadratic problems," Nonlinear Analysis: Theory, Methods \& Applications, vol. 75, no. 2, pp. 932-950, 2012.

[20] D. R. Poulsen, Stability and control on stochastic time scales, Ph.D thesis, Baylor University, Waco, TX, USA, 2015.

[21] D. R. Poulsen, J. M. Davis, and I. A. Gravagne, "Optimal control on stochastic time scales," IFAC-PapersOnLine, vol. 50, no. 1, pp. 14861-14866, 2017.

[22] S. Chen, X. Li, and X. Y. Zhou, "Stochastic linear quadratic regulators with indefinite control weight costs," SIAM Journal on Control and Optimization, vol. 36, no. 5, pp. 1685-1702, 1998.

[23] S. Chen and X. Y. Zhou, "Stochastic linear quadratic regulators with indefinite control weight costs. II," SIAM Journal on Control and Optimization, vol. 39, no. 4, pp. 1065-1081, 2000.

[24] Y. Zhu and G. Jia, "Linear feedback of mean-field stochastic linear quadratic optimal control problems on time scales," Mathematical Problems in Engineering, vol. 2020, Article ID 8051918, 11 pages, 2020.

[25] S. Boyd, L. El Ghaoui, E. Feron, and V. Balakrishnan, Linear Matrix Inequality in Systems and Control Theory, Siam Frontier Series, SIAM, Philadelphia, PA, USA, 1994.

[26] D. Grow and S. Sanyal, "The quadratic variation of Brownian motion on a time scale," Statistics \& Probability Letters, vol. 82, no. 9, pp. 1677-1680, 2012.

[27] S. Sanyal, Stochastic Dynamic Equations, Ph.D thesis, Missouri University of Science and Technology, Rolla, MO, USA, 2008. 
[28] A. A. Stoorvogel and A. J. T. M. Weeren, "The discrete-time Riccati equation related to the $\mathrm{H} /$ sub $\infty /$ control problem," IEEE Transactions on Automatic Control, vol. 39, no. 3, pp. 686-691, 1994.

[29] M. A. Rami, X. Chen, J. B. Moore, and X. Y. Zhou, "Solvability and asymptotic behavior of generalized Riccati equations arising in indefinite stochastic LQ controls," IEEE Transactions on Automatic Control, vol. 46, no. 3, pp. 428-440, 2001.

[30] R. P. Agarwal and M. Bohner, "Basic calculus on time scales and some of its applications," Results in Mathematics, vol. 35, no. $1-2$, pp. 3-22, 1999. 